\title{
Diversity-Enabled and Power-Efficient Transceiver Designs for Peak-Power-Limited SIMO-OFDM Systems
}

\author{
Qijia Liu, ${ }^{1}$ Robert J. Baxley, ${ }^{2}$ Xiaoli Ma, ${ }^{1}$ and G. Tong Zhou ${ }^{1}$ \\ ${ }^{1}$ School of Electrical and Computer Engineering, Georgia Institute of Technology, 777 Atlantic Drive, Atlanta, GA 30332-0250, USA \\ ${ }^{2}$ Information Technology and Telecommunications Laboratory (ITTL), Georgia Tech Research Institute, 250 14th Street, NW, \\ Atlanta, GA 30318, USA
}

Correspondence should be addressed to Qijia Liu, qliu6@mail.gatech.edu

Received 12 November 2009; Accepted 4 March 2010

Academic Editor: Cihan Tepedelenlioğlu

Copyright (C) 2010 Qijia Liu et al. This is an open access article distributed under the Creative Commons Attribution License, which permits unrestricted use, distribution, and reproduction in any medium, provided the original work is properly cited.

\begin{abstract}
Orthogonal frequency division multiplexing (OFDM) has been widely adopted for high data rate wireless transmissions. By deploying multiple receiving antennas, single-input multiple-output- (SIMO-) OFDM can further enhance the performance with spatial diversity. However, due to the large dynamic range of OFDM signals and the nonlinear nature of analog components, it is pragmatic to model the transmitter with a peak-power constraint. A natural question to ask is whether SIMO-OFDM transmissions can still enjoy the antenna diversity in this case. In this paper, the effect of the peak-power limit on the error performance of uncoded SIMO-OFDM systems is studied. In the case that the receiver has no information about the transmitter nonlinearity, we show that full antenna diversity can still be collected by carefully designing the transmitters, while the receiver performs a maximum ratio combining (MRC) method which is implemented the same as that in the average power constrained case. On the other hand, when the receiver has perfect knowledge of the peak-power-limited transmitter nonlinearity, zero-forcing (ZF) equalizer is able to collect full antenna diversity. In addition, an iterative method on joint MRC and clipping mitigation is proposed to achieve high performance with low complexity.
\end{abstract}

\section{Introduction}

Orthogonal frequency division multiplexing (OFDM) has been adopted by various modern communication standards because of its high spectral efficiency and low complexity in combating frequency-selective fading effects $[1,2]$. Equipped with multiple antennas, OFDM systems can further enhance the performance by collecting spatial diversity [3]. Thus, multiple-input multiple-output (MIMO) OFDM transmission has been adopted by several communication standards and becomes a strong candidate for future cellular systems [4].

However, OFDM experiences certain implementation challenges due to the large dynamic range of its signal waveforms, which is usually measured by the peak-toaverage power ratio (PAR) [5]. Large PAR values may lead to low power efficiency or severe nonlinear distortions which decrease system performance. It is possible to back-off (i.e., scale down) the waveform so that distortions are less likely, but this comes at the cost of the reduced transmission power efficiency. Conversely, although the signal power can be boosted by reducing the amount of back-off, nonlinear distortions will inevitably be increased. Power efficiency and nonlinear distortions are thus conflicting metrics that must be balanced. There has been extensive research on improving the transmission power efficiency with constraints on nonlinear distortions in single-input single-output (SISO) OFDM channels (see e.g., [6]).

In light of the power efficiency and nonlinear distortion considerations, the error performance of OFDM systems with peak-power-limited power amplifier (PA) should be investigated. To quantify the error performance of wireless transmissions over fading channels, two parameters are usually used: diversity order and coding gain (see e.g., $[7,8]$ ). The diversity order describes how fast the error probability decays with signal-to-noise ratio (SNR), while the coding 
gain measures the error performance gap among different schemes when they have the same diversity. Thus, diversityenabled transceivers have well-appreciated merits. For singleantenna OFDM systems with clipping at the transmitter, the approximated symbol error rate (SER) has been derived for maximum-likelihood sequence detection (MLSD) [9]. The results show that the clipping nonlinearity leads to a certain (may not be full) multipath diversity order over frequencyselective Rayleigh fading channels. However, MLSD requires near exponential complexity to collect some diversity gain. When the number of subcarriers is large which is usually the case in current standards, the complexity of MLSD is prohibitive. In such a case, the OFDM system loses its advantage as a simple equalizer which may reduce its practical applicability.

In this paper, we are interested in low-complexity diversity-enabled transceiver design over peak-power-limited channels. Instead of the multipath diversity, we focus on the antenna diversity from multiple antennas deployed at the receiver (i.e., single-input multiple-output (SIMO) channels). When OFDM signals are linearly transmitted, linear equalizers are sufficient to collect the antenna diversity by optimally combining the multiple faded replicas of the same information bearing signal $[10,11]$. However, to the best of our knowledge, the question of whether and how the peakpower-limited SIMO-OFDM system can still enjoy antenna diversity with linear equalizers has not been addressed in the literature. A few iterative methods to reconstruct the clipped OFDM signals in multiple-antenna systems have been proposed in [12-14] based on the assumption that the receiver knows the transmitter nonlinearity. However, the diversity gain has not been quantitatively analyzed.

This paper focuses on error performance analysis for SIMO-OFDM systems over peak-power-limited channels. Several low-complexity transceiver designs are proposed to collect the antenna diversity and near maximum-likelihood (ML) SER performance is achieved.

The rest of the paper is organized as follows. The OFDM system and SIMO channel models are described in Section 2. In Section 3, the diversity combining methods for linear SIMO channels are briefly reviewed. The transceiver designs over the peak-power-limited SIMO channels are mainly discussed in Sections 4 and 5 based on different a priori information requirements. Numerical results are shown in Section 6. Finally, conclusions are drawn in Section 7.

Notation. Throughout this paper, we use lower-case and upper-case bold face letters for column vectors and matrices, respectively. Their elements are denoted in italic with subindices. ${ }^{*}$ denotes conjugate, ${ }^{T}$ transpose, and ${ }^{H}$ Hermitian. Let blackboard bold letters represent number sets, then $\mathbb{A}^{m \times n}$ stands for an $m \times n$ matrix whose elements belong to a number set $\mathbb{A}$. In particular, we use $\mathbb{C}$ to represent the set of all complex numbers. $\|\mathbf{x}\|_{\ell}$ stands for the $\ell$ th norm of vector x. $\mathbf{0}_{l}$ is an $l$-by- 1 vector with all zero entries and $\mathbf{I}_{l \times l}$ is an $l$ by- $l$ identity matrix. $\operatorname{diag}(\mathbf{x})$ denotes a diagonal matrix with vector $\mathbf{x}$ on its diagonal and $\operatorname{tr}(\cdot)$ stands for the trace of a matrix. Additionally, $E_{x}[\cdot]$ is used for the expectation over a random variable $x$.

\section{System Model}

In an uncoded OFDM system, data are transmitted on $N$ orthogonal subcarriers. The frequency-domain OFDM symbols are denoted as $\mathbf{s}=\left[s_{0}, \ldots, s_{N-1}\right]^{T} \in \mathbb{S}^{N \times 1}$ where $s_{k}$ 's are drawn from an ideal constellation $\mathbb{S}$. For notational simplicity, equal power allocation among subcarriers is assumed in this paper, but the proposed methods can be generalized with minor modifications. Prior to cyclic extension (which does not impact the signal dynamic range [5]), the $L$-times oversampled time-domain waveform can be obtained from the $L N$-point inverse fast Fourier transform (IFFT) operation, that is, (c.f. [5])

$$
\mathbf{x}=\left[x_{0}, \ldots, x_{L N-1}\right]^{T}=\mathbf{F}^{H} \mathbf{s} \in \mathbb{C}^{L N \times 1},
$$

where $\mathbf{F}$ is the $N \times L N$ oversampling FFT matrix formed by retaining only the first $N$ rows of a full FFT matrix whose $(m+1, n+1)$ th entry is $(1 / \sqrt{L N}) e^{-j 2 \pi m n /(L N)}$. Since this FFT operation is unitary, we have $E_{\mathbf{s}}\left[(1 / N)\|\mathbf{s}\|_{2}^{2}\right]=$ $E_{\mathbf{x}}\left[(1 / L N)\|\mathbf{x}\|_{2}^{2}\right] \triangleq \sigma_{s}^{2}$.

To characterize the dynamic range of the OFDM signal, the peak-to-average power ratio (PAR) for each OFDM symbol is defined as

$$
\operatorname{PAR}(\mathbf{x})=\frac{\|\mathbf{x}\|_{\infty}^{2}}{(1 / L N)\|\mathbf{x}\|_{2}^{2}} .
$$

There is a peak-power-limited PA with output peakpower limit $P_{\text {peak }}$ before the signal is transmitted. Here we assume an ideal linear class-A PA, which implies that the time-domain output signal $y_{n}=g\left(x_{n}\right)$ is characterized by [5, Chapter 3]

$$
y_{n}=g\left(x_{n}\right)= \begin{cases}x_{n}, & \left|x_{n}\right| \leq \sqrt{P_{\text {peak }}}, \\ \sqrt{P_{\text {peak }}} e^{j}<x_{n}, & \left|x_{n}\right|>\sqrt{P_{\text {peak }}}\end{cases}
$$

where $\angle x$ denotes the phase of a complex variable $x$. Without loss of generality, unit gain is assumed for the PA linear region. The input back-off (IBO) is defined as $\mathrm{IBO}=$ $P_{\text {peak }} / \sigma_{s}^{2}$. Clipping occurs when $\operatorname{PAR}(\mathbf{x})>\mathrm{IBO}$.

The frequency-domain symbol corresponding to the in-band subcarriers can be obtained from $\mathbf{y}=\left[y_{0}, \ldots, y_{L N-1}\right]^{T} \triangleq g(\mathbf{x})$ as

$$
\overline{\mathbf{s}}=\mathbf{F y} .
$$

Notice that, by digital clipping and filtering methods, outof-band spectral regrowth can be constrained according to certain spectral mask or totally eliminated $[15,16]$. In this case, the following analysis still holds valid and the proposed methods can be modified accordingly by treating the clipping and filtering as a deterministic nonlinear process.

The receiver is equipped with $N_{r}$ uncorrelated receiving antennas. After removing the cyclic extension and performing the FFT, the received signal in frequency-selective Rayleigh fading channels is

$$
\mathbf{r}=\left[\mathbf{r}_{1}^{T}, \ldots, \mathbf{r}_{N_{r}}^{T}\right]^{T}=\mathbf{H} \overline{\mathbf{s}}+\mathbf{w}
$$


where $\mathbf{r}_{i}$ denotes the OFDM symbol received on the $i$ th antenna. $\mathbf{H}=\left[\mathbf{H}_{1}, \ldots, \mathbf{H}_{N_{r}}\right]^{T}, \mathbf{H}_{i}=\operatorname{diag}\left(\left[H_{i, 0}, \ldots, H_{i, N-1}\right]\right)$, and $H_{i, k}(0 \leq k \leq N-1)$ is the channel frequency response of the $k$ th in-band subcarrier on the $i$ th receiving antenna. In addition, $\mathbf{w}=\left[\mathbf{w}_{1}^{T}, \ldots, \mathbf{w}_{N_{r}}^{T}\right]^{T}$ and $\mathbf{w}_{i}=\left[w_{i, 0}, \ldots, w_{i, N-1}\right]^{T}$ where $w_{i, k}(0 \leq k \leq N-1)$ consists of the circularly complex white Gaussian noise with variance $\sigma_{w}^{2}$.

In this paper, we study the symbol error rate (SER) in peak-power-limited SIMO fading channels. First, we give some definitions.

Definition 1 (PSNR). For the peak-power-limited PA, the peak-signal-to-noise ratio (PSNR) is used to compare the PA power consumption and the channel noise level, that is,

$$
\operatorname{PSNR}=\frac{P_{\text {peak }}}{\sigma_{w}^{2}}=\mathrm{IBO} \cdot \frac{\sigma_{s}^{2}}{\sigma_{w}^{2}},
$$

which is the product of IBO and the usual average signal-tonoise ratio (SNR), that is, $\mathrm{SNR}=\sigma_{s}^{2} / \sigma_{w}^{2}$.

Definition 2 (Diversity gain). Suppose that $P_{s}$ (PSNR) is the average SER for a certain peak-power-limited system as a function of PSNR. We define the diversity gain $G_{d}$ as

$$
G_{d}=\lim _{\mathrm{PSNR} \rightarrow \infty}-\frac{\log P_{s}(\mathrm{PSNR})}{\log \mathrm{PSNR}} .
$$

Unlike linear channels, the SER and the diversity gain are defined in terms of PSNR in peak-power-limited channels. For certain transmitters with a given $P_{\text {peak }}$, the diversity gain describes how fast the SER decays with decreasing channel noise power.

\section{Diversity Combining in Linear SIMO Channels}

For linear SIMO channels, several diversity combining techniques are available to achieve the antenna diversity [10], for example, maximal ratio combining (MRC) and selective combining (SC). Before discussing the peak-power-limited case, we briefly review the MRC method for the linear SIMOOFDM channel.

Suppose that the receiver has perfect channel knowledge. Without the peak-power limit, the received signal of (5) becomes $\mathbf{r}=\mathbf{H s}+\mathbf{w}$. The MRC method chooses the $N_{r} N \times N$ coefficient matrix $\mathbf{C}=\left[\mathbf{c}_{0}, \ldots, \mathbf{c}_{N-1}\right]$ to combine the received signal, where $\boldsymbol{c}_{k} \in \mathbb{C}^{N_{r} N \times 1}$ is the $k$ th column of $\mathbf{C}$. The estimate of $\mathbf{s}$ is thus given as

$$
\widetilde{\mathbf{s}}=\mathbf{C}^{T} \mathbf{H s}+\mathbf{C}^{T} \mathbf{w} .
$$

To maximize the postprocessing (received) SNR for an uncoded OFDM system, the optimal weights can be shown as [10]

$$
\mathbf{c}_{k}=\frac{\mathbf{h}_{k}^{*}}{\mathbf{h}_{k}^{H} \mathbf{h}_{k}},
$$

where $\mathbf{h}_{k}$ is the $k$ th column of $\mathbf{H}$, that is, $\mathbf{H}=\left[\mathbf{h}_{0}, \ldots, \mathbf{h}_{N-1}\right]$. The corresponding received SNR is $\mathbf{h}_{k}^{H} \mathbf{h}_{k}$ SNR. In the end, the decision $\widehat{\mathbf{s}}$ is obtained by hard decoding on $\widetilde{\mathbf{s}}$, denoted as $\widehat{\mathbf{s}}=\langle\widetilde{\mathbf{s}}\rangle$.

Therefore, for uncoded SIMO-OFDM, MRC is essentially the zero-forcing ( $\mathrm{ZF}$ ) and also the maximumlikelihood (ML) equalizer in the linear SIMO channel with Gaussian noise, that is, $\mathbf{C}^{T}=\mathbf{H}^{\dagger}$ where $\mathbf{H}^{\dagger}=\left(\mathbf{H}^{H} \mathbf{H}\right)^{-1} \mathbf{H}^{H}$ is the Moore-Penrose pseudo-inverse of $\mathbf{H}$ [17]. When an $M$ ary QAM constellation is used, the average SER over SIMO Rayleigh fading channels is [18]

$$
P_{s}(\mathrm{SNR})=\frac{4 \sqrt{M}-4}{\sqrt{M}}\left(\frac{1-\mu}{2}\right)^{N_{r}} \sum_{i=0}^{N_{r}-1} \frac{\left(N_{r}-1+i\right) !}{i !\left(N_{r}-1\right) !}\left(\frac{1+\mu}{2}\right)^{i},
$$

where $\mu=(1+2(M-1) / 3 \mathrm{SNR})^{-1 / 2}$. It is ready to show that

$$
\lim _{\mathrm{SNR} \rightarrow \infty}-\frac{\log P_{s}(\mathrm{SNR})}{\log \mathrm{SNR}}=N_{r}
$$

that is, MRC collects full antenna diversity. From the existing literature, however, it is not clear yet whether (and if so, how) full antenna diversity can be achieved in the presence of the peak-power constraint. We address this open question in the following sections.

\section{Transparent Receivers: A Statistical Model}

By "transparent" we mean that the receivers have no information about the transmitter nonlinearities. In this case, no receiver-side cooperation is expected. The nonlinear distortion noise has to be dealt with in the same way as the uncorrelated Gaussian channel noise. Therefore, the following statistical model is introduced at first to quantify the clipping noise.

Definition 3 (Statistical model). According to Bussgang's theorem [19], the clipped waveform $y_{n}$ in (3) can be decomposed into a linear term $\alpha x_{n}$ plus a statistically uncorrelated distortion term $u_{n}$, that is,

$$
y_{n}=\alpha x_{n}+u_{n}
$$

where $\alpha=E\left[x_{n}^{*} y_{n}\right] / E\left[\left|x_{n}\right|^{2}\right]$ is chosen so that the signal $x_{n}$ and the nonlinear distortion noise $u_{n}$ are uncorrelated, that is, $E\left[x_{n}^{*} u_{n}\right]=0$. Because clipping causes $\left|y_{n}\right| \leq\left|x_{n}\right|$, we have $|\alpha| \leq 1$ and thus the effective signal power is reduced. The distortion noise power is $\sigma_{u}^{2}=E\left[\left|y_{n}\right|^{2}\right]-|\alpha|^{2} E\left[\left|x_{n}\right|^{2}\right]$. The received frequency-domain symbol is thus given by

$$
\mathbf{r}=\mathbf{H}^{\prime} \mathbf{s}+\mathbf{H v}+\mathbf{w}
$$

where $\mathbf{H}^{\prime}=\alpha \mathbf{H}$ is the equivalent channel frequency response. The frequency-domain nonlinear distortion noise can be found as $\mathbf{v}=$ Fu with power $\sigma_{v}^{2} \triangleq E\left[(1 / N)\|\mathbf{v}\|_{2}^{2}\right]=$ $E\left[(1 / L N)\|\mathbf{u}\|_{2}^{2}\right]=\sigma_{u}^{2}$.

In the presence of distortion noise, signal-to-noise-anddistortion ratio (SNDR) should be used to incorporate both the signal power attenuation and nonlinear distortions, 
and characterize the overall SER performance in the given channel [20]. Based on the statistical model, the postprocessing SNDR of the $k$ th subcarrier is given as

$$
\mathrm{SNDR}_{k}=\frac{|\alpha|^{2}\left|\mathbf{c}_{k}^{T} \mathbf{h}_{k}\right|^{2} \sigma_{s}^{2}}{\left|\mathbf{c}_{k}^{T} \mathbf{h}_{k}\right|^{2} \sigma_{v}^{2}+\mathbf{c}_{k}^{H} \mathbf{c}_{k} \sigma_{w}^{2}}, \quad k \in\{0, \ldots, N-1\} .
$$

To maximize the SNDR, the MRC weights are given in the following proposition.

Proposition 1. For transparent receivers that have no information about the transmitter nonlinearity, the optimal MRC weights are given by $\mathbf{C}$ whose $k$ th column is $\mathbf{c}_{k}=\mathbf{h}_{k}^{\prime *} / \mathbf{h}_{k}^{\prime H} \mathbf{h}_{k}^{\prime}$, where $\mathbf{h}_{k}^{\prime}=\alpha \mathbf{h}_{k}(k \in\{0, \ldots, N-1\})$.

Proof. See Appendix A.

At first, it appears that the transparent receiver has to know $\alpha$ in order to acquire $\mathbf{C}$, which is inconsistent with the "transparent" definition. In fact, for OFDM systems with embedded pilot subcarriers, since the pilot signals are also attenuated by $\alpha, \mathbf{H}^{\prime}=\alpha \mathbf{H}$ is the effective channel response which is acquired by channel estimation at the receiver. Therefore, transparent receivers do not need to know $\alpha$ aforehand and the SNDR-maximizing combining weights can be used to achieve the best error performance.

Unlike the linear case, using the optimal MRC weights at the receiver may not guarantee full antenna diversity. The necessary and sufficient condition for achieving the antenna diversity gain with transparent receivers is given as follows.

Proposition 2. For OFDM transmitters with a fixed peakpower limit, the transparent receiver is able to achieve full antenna diversity if and only if the distortion noise vanishes as the PSNR increases.

Proof. See Appendix B.

Proposition 2 demonstrates that the distortions at the transmitter have to be controlled in order to achieve the antenna diversity with transparent receivers. The corresponding system diagram is shown in Figure 1(a). In the following, we give some examples to illustrate the design of the diversity-enabled peak-power-limited OFDM transmitter. The performance will be verified by simulations in Section 6.

Example 1 (Constant clipping). When a constant IBO is maintained, clipping occurs if the PAR of an OFDM symbol exceeds the IBO. It implies that $|\alpha|<1$ and $\sigma_{v}^{2}>0$ for the statistical model in (12). Therefore, no antenna diversity can be achieved with transparent receivers. In fact, as indicated in Appendix B, error floor should be observed.

Example 2 (Piece-wise linear scaling). The piece-wise linear scaling (PWLS) method is a simple way to guarantee that no distortion happens with the soft-limiter PA [21]. It is realized

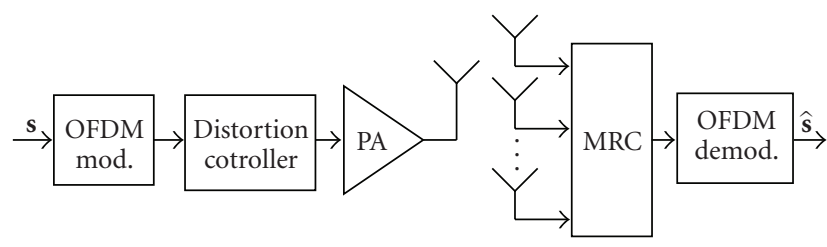

(a) The system structure with transparent receivers

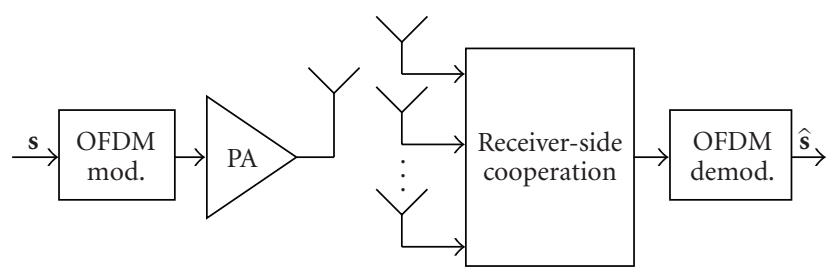

(b) The system structure with receiver-side cooperations

FIGURE 1: Transceiver block diagrams.

by multiplying a symbol-wise gain to each OFDM symbol before passing it to the PA, namely,

$$
\overline{\mathbf{x}}=\frac{\sqrt{P_{\text {peak }}}}{\|\mathbf{x}\|_{\infty}} \mathbf{x} .
$$

Because $\left|\bar{x}_{n}\right|^{2} \leq P_{\text {peak }}$ so that clipping never occurs, we have $g(\overline{\mathbf{x}})=\overline{\mathbf{x}}$ and $\overline{\mathbf{s}}=\left(\sqrt{P_{\text {peak }}} /\|\mathbf{x}\|_{\infty}\right)$ s in (3) and (4). The symbol-wise gain will not affect the demodulation because it is essentially a part of the channel and can be recovered by receivers with pilot-aided channel estimation.

Proposition 2 indicates that full antenna diversity can be achieved with PWLS. Owing to the linear transmission, the postprocessing SNDR becomes

$$
\begin{aligned}
\mathrm{SNDR}_{k} & =\mathbf{h}_{k}^{H} \mathbf{h}_{k} \frac{E\left[\left(P_{\text {peak }} /\|\mathbf{x}\|_{\infty}^{2}\right)\left|s_{k}\right|^{2}\right]}{\sigma_{w}^{2}} \\
& =\mathbf{h}_{k}^{H} \mathbf{h}_{k} \frac{P_{\text {peak }} E\left[\|\mathbf{x}\|_{2}^{2} / L N\|\mathbf{x}\|_{\infty}^{2}\right]}{\sigma_{w}^{2}} \\
& =\mathbf{h}_{k}^{H} \mathbf{h}_{k} \cdot \operatorname{PSNR} \cdot E\left[\operatorname{PAR}(\mathbf{x})^{-1}\right],
\end{aligned}
$$

which is inversely proportional to the harmonic mean of the PAR. Still, low power efficiency and small coding gain may result due to the large PAR of OFDM signals. A number of distortionless methods have been proposed to reduce the PAR of OFDM signals, for example, coding [22], selected mapping [23], and tone reservation [5]. They can be combined with PWLS and improve the coding gain at the cost of implementation complexity, spectral efficiency and/or receiver-side cooperation.

Example 3 (Optimal clipping). When the PSNR is known at the transmitter, an optimal amount of clipping distortion can be methodically introduced to improve the error performance for transparent receivers $[20,24]$. 
Instead of the original OFDM waveform, the following signal is input to the PA:

$$
\bar{x}_{n}= \begin{cases}\frac{\sqrt{P_{\text {peak }}}}{\eta \sigma_{s}} x_{n}, & \frac{\left|x_{n}\right|}{\sigma_{s}}<\eta, \\ \sqrt{P_{\text {peak }}} e^{j<x_{n}}, & \frac{\left|x_{n}\right|}{\sigma_{s}} \geq \eta,\end{cases}
$$

where $\eta \geq 0$ is called the clipping threshold [24]. Because $\left|\bar{x}_{n}\right|^{2} \leq P_{\text {peak }}$, the PA output has $\mathbf{y}=\overline{\mathbf{x}}$. Accordingly, the Bussgang parameters $\alpha$ and $\sigma_{v}^{2}$ in (12) can be numerically determined for different $\eta$ 's. Then, the postprocessing SNDR for the optimal clipping can be found as

$$
\operatorname{SNDR}_{k}=\frac{\mathbf{h}_{k}^{H} \mathbf{h}_{k}|\alpha|^{2} P_{\text {peak }}}{\eta^{2} \mathbf{h}_{k}^{H} \mathbf{h}_{k} \sigma_{v}^{2}+\eta^{2} \sigma_{w}^{2}} .
$$

If the channel noise level $\sigma_{\mathrm{w}}^{2}$ (or PSNR) is known at the transmitter, the optimal clipping threshold can be determined to minimize the average SER, that is,

$$
\eta^{\circ}=\arg \min _{\eta} \sum_{k=0}^{N-1} E_{\mathbf{h}_{k}}\left[p\left(\mathrm{SNDR}_{k}\right)\right]
$$

where $p\left(\mathrm{SNDR}_{k}\right) \approx((4 \sqrt{M}-4) / \sqrt{M}) Q\left(\sqrt{3 \mathrm{SNDR}_{k} /(M-1)}\right)$ is the SER for $M$-ary QAM constellations and $Q(x)=$ $\operatorname{erfc}(x / \sqrt{2}) / 2$ [7, page 278]. When the OFDM waveform is approximated as a complex Gaussian random variable, a numerical method to solve for $\eta^{\circ}$ can be found in [24].

Unlike PWLS which is trying to avoid any clipping, the optimum clipping method maximizes the SNDR in (18) for a given PSNR. In the high PSNR region, a large $\eta^{\circ}$ is yielded in which case $|\alpha| \rightarrow 1$ and $\sigma_{v}^{2} \rightarrow 0$ [24]. Thus, full antenna diversity is sustained according to Proposition 2. On the other hand, in the low PSNR region, some distortion is introduced to achieve a more desired tradeoff with the increase in signal power so that the error performance is optimized. Therefore, the optimal clipping method can achieve a better coding gain while maintaining the full antenna diversity for transparent receivers.

\section{Transmitter Nonlinearity Known at the Receiver: A Deterministic Model}

Instead of a random process, the clipping distortion, based on the PA model in (3), is a deterministic function of the data. When the receiver knows or estimates a priori the transmitter nonlinearity, it can exploit the deterministic nature of the clipping process for better performance [25]. In this case, receiver-side cooperation can be adopted to achieve antenna diversity with nondiminishing distortion noise at the transmitter. The corresponding system diagram is given in Figure 1(b).

In order to design the receiver-side cooperation, we first establish a deterministic model to characterize the clipping process.
Definition 4 (Deterministic model). After clipping, the frequency-domain OFDM symbol in (4) can be represented by the following deterministic matrix operation $[25,26]$

$$
\overline{\mathbf{s}}=\mathbf{F} \mathbf{\Lambda} \mathbf{F}^{H} \mathbf{s}=\mathbf{s}+\mathbf{d},
$$

where

$$
\boldsymbol{\Lambda}=\operatorname{diag}\left(\left[\min \left(\frac{\sqrt{P_{\text {peak }}}}{\left|x_{0}\right|}, 1\right), \ldots, \min \left(\frac{\sqrt{P_{\text {peak }}}}{\left|x_{L N-1}\right|}, 1\right)\right]\right)
$$

is the function of $\mathbf{s}$ and $\mathbf{d}=\mathbf{F}(g(\mathbf{x})-\mathbf{x})$ is the frequencydomain representation of the deterministic clipping noise.

As proven in $[9,27]$, when $\mathrm{IBO} \geq 3 \pi(\sqrt{M}-3)^{2} / 8(M-$ 1) for $M$-ary $\operatorname{QAM}(M \geq 16)$ and when the MLSD receiver is used, clipping the Nyquist-sampling OFDM signal only causes a constant SNR loss on the SER performance. Therefore, with constant clipping, the effective transmit SNR becomes SNR $\approx \Delta(\mathrm{IBO}) \mathrm{PSNR} / \mathrm{IBO}$, where $\Delta(\mathrm{IBO}) \approx 1-$ $e^{-\mathrm{IBO}}+(1 / 2) \mathrm{IBO} \int_{\mathrm{IBO}}^{\infty} e^{-t} / t d t \leq 1$. Plugging this effective SNR into (10), the average SER of MLSD in flat Rayleigh fading SIMO channels is given by

$$
P_{\mathrm{MLSD}}(\mathrm{PSNR}, \mathrm{IBO}) \approx P_{s}\left(\frac{\Delta(\mathrm{IBO}) \cdot \mathrm{PSNR}}{\mathrm{IBO}}\right) .
$$

Although clipping was also shown to enable certain multipath diversity in frequency-selective fading channels [9], we focus on antenna diversity in this paper. In addition, the SER performance for clipping and filtering oversampled OFDM signals was shown to be well approximated by that of the Nyquist sampling in SISO fading channels [9]. This approximation remains for the SIMO channel case. Therefore, the average SER for general SIMO fading channels can be approximated by (22), which is referred as the MLSD bound in accordance with [9]. Again, full antenna diversity can be verified similar to (B.4) in Appendix B.

However, MLSD receivers have exponential complexity, which is not practical for implementations especially for a large number of subcarriers. Instead, linear equalizers are usually used as low-complexity solutions, but do not necessarily offer the same diversity gains as MLSD [17]. For the received signal in (5), if $\boldsymbol{\Lambda}$ is known at the receiver, the ZF equalizer is given as

$$
\widetilde{\mathbf{s}}_{\mathrm{zf}}=\mathscr{H}^{\dagger} \mathbf{r}=\mathbf{s}+\mathscr{H}^{\dagger} \mathbf{w},
$$

where $\mathscr{H}=\operatorname{HF} \Lambda \mathbf{F}^{H}$. In the following, we first quantify the diversity order collected by the ZF equalizer when $\Lambda$ is known. Then, an iterative method will be proposed to jointly estimate both $\boldsymbol{\Lambda}$ and $\boldsymbol{s}$ and realize the ZF equalizer in the absence of a priori knowledge about $\Lambda$.

Proposition 3. For clipped OFDM signals transmitted through SIMO fading channels with $N_{r}$ receiving antennas, if the receiver has perfect knowledge of the $\boldsymbol{\Lambda}$ given in (21), the diversity order collected by the ZF equalizer is $N_{r}$. 
Proof. See Appendix C.

Proposition 3 states that ZF equalizers can achieve full antenna diversity if the clipping-based matrix $\boldsymbol{\Lambda}$ is known or can be estimated at the receiver. It also indicates that in frequency-selective fading channels, ZF equalizers are not able to collect any multipath diversity. It is the compromise that low-complexity solutions have to make. The same fact was previously observed in [9] without proof. It is also worth mentioning that, unlike the linear case in Section 3, MRC is no longer the same as the ZF equalizer in the presence of clipping.

Although $\boldsymbol{\Lambda}$ is a function of the data $\mathbf{s}$ and cannot be known a priori at the receiver, the following recursive method can jointly estimate $\boldsymbol{\Lambda}$ and s. The transmitter peak-power limit $P_{\text {peak }}$ is assumed available at the receiver. Based on decision feedback, the proposed iterative method can be summarized in three steps:

$$
\begin{gathered}
\hat{\mathbf{s}}^{(q)}=\left\langle\left(\mathbf{H F} \hat{\Lambda}^{(q-1)} \mathbf{F}^{H}\right)^{\dagger} \mathbf{r}\right\rangle \\
\hat{\mathbf{x}}^{(q)}=\mathbf{F}^{H} \hat{\mathbf{s}}^{(q)}, \\
\hat{\Lambda}^{(q)}=\operatorname{diag}\left(\left[\min \left(\frac{\sqrt{P_{\text {peak }}}}{\left|\hat{x}_{0}^{(q)}\right|}, 1\right), \ldots, \min \left(\frac{\sqrt{P_{\text {peak }}}}{\left|\hat{x}_{L N-1}^{(q)}\right|}, 1\right)\right]\right),
\end{gathered}
$$

where ${ }^{\wedge}$ denotes the estimate for the corresponding variable and the superscript $(\cdot)^{(q)}$ stands for the iteration index. As the initialization, $\hat{\Lambda}^{(0)}=\mathbf{I}_{L N \times L N}$.

Calculating the pseudoinverse in (24) may require high computational complexity, but it can be further simplified as $\left(\mathbf{H F} \mathbf{\Lambda} \mathbf{F}^{H}\right)^{\dagger}=\left(\mathbf{F} \boldsymbol{\Lambda} \mathbf{F}^{H}\right)^{-1} \mathbf{H}^{\dagger}$, where $\mathbf{H}^{\dagger}=\mathbf{C}^{T}$ (i.e., the MRC weights), because of the full column ranks of $\mathbf{F} \mathbf{\Lambda} \mathbf{F}^{H}$ and $\mathbf{H}$ [28]. Moreover, the inverse of $\mathbf{F} \boldsymbol{\Lambda} \mathbf{F}^{H}$ can be avoided because

$$
\left(\mathbf{F} \boldsymbol{\Lambda} \mathbf{F}^{H}\right)^{-1}=\mathbf{I}-\mathbf{F}(\boldsymbol{\Lambda}-\mathbf{I}) \mathbf{F}^{H}\left(\mathbf{F} \boldsymbol{\Lambda} \mathbf{F}^{H}\right)^{-1}
$$

In each iteration, the estimate of $\mathbf{s}$ can be recursively updated as

$$
\hat{\mathbf{s}}^{(q)}=\left\langle\mathbf{H}^{\dagger} \mathbf{r}-\mathbf{F}\left(\hat{\boldsymbol{\Lambda}}^{(q-1)}-\mathbf{I}\right) \mathbf{F}^{H} \widehat{\mathbf{s}}^{(q-1)}\right\rangle .
$$

Further, because $\mathbf{F}(\boldsymbol{\Lambda}-\mathbf{I}) \mathbf{F}^{H} \mathbf{s}=\mathbf{d}$, the clipping noise can be estimated (i.e., $\widehat{\mathbf{d}}=\mathbf{F}(g(\widehat{\mathbf{x}})-\widehat{\mathbf{x}}))$ to avoid the FFT, IFFT, and matrix inverse operations for $\left(\mathbf{F} \Lambda \mathbf{F}^{H}\right)^{-1}$. Therefore, the iterative method in (31) is equivalent to the following lowcomplexity method, starting with $q=1$ and $\widehat{\mathbf{d}}^{(0)}=\mathbf{0}_{N}$ :

$$
\begin{gathered}
\hat{\mathbf{s}}^{(q)}=\left\langle\mathbf{C}^{T} \mathbf{r}-\hat{\mathbf{d}}^{(q-1)}\right\rangle, \\
\hat{\mathbf{x}}^{(q)}=\mathbf{F}^{H} \hat{\mathbf{s}}^{(q)}, \\
\hat{\mathbf{d}}^{(q)}=\mathbf{F}\left(g\left(\hat{\mathbf{x}}^{(q)}\right)-\widehat{\mathbf{x}}^{(q)}\right) .
\end{gathered}
$$

We refer to it as the joint MRC and clipping mitigation method. Its complexity is dominated by one pair of
FFT/IFFT operations per iteration and on the order of $\mathcal{O}(N \log N)$.

The mean square error (MSE) of the estimate of $\hat{\mathbf{d}}^{(q)}$ can be defined as

$$
\operatorname{MSE}_{\mathbf{d}}^{(q)}=E\left[\left\|\mathbf{d}-\hat{\mathbf{d}}^{(q)}\right\|_{2}^{2}\right]
$$

$\operatorname{MSE}_{\mathbf{d}}^{(q)}$ is decreasing quickly, especially in the high PSNR region, which will be shown in Section 6. As a result, the joint estimation method can empirically approach the ideal case of ZF equalizers. Acting as the receiver-side cooperation as plotted in Figure 1(b), it can collect full antenna diversity with constant clipping at the transmitter.

Two more remarks about the use of the joint MRC and clipping mitigation method are now in order.

Remark 1. The smaller the IBO, the larger the ratio PSNR/IBO for a fixed PSNR. Meanwhile, however, $\triangle$ (IBO) in (22) decreases along with the decrease of IBO. Therefore, an optimal IBO exists with respect to the SER performance, which can be found as

$$
\left.\mathrm{IBO}^{\circ}\right|_{\mathrm{PSNR}}=\arg \min _{\mathrm{IBO}} P_{\text {sim }}\left(\mathrm{PSNR} \mid \mathrm{IBO}, N_{r}\right),
$$

where $P_{\text {sim }}(\cdot)$ denotes the simulated average SER performance for the joint MRC and clipping mitigation method.

Remark 2. The proposed method can be regarded as an extension to the iterative quasi-ML clipping estimation method [29], which was designed for SISO-OFDM systems. However, the quasi-ML clipping estimation method provides poor error performance in fading channels, which will be shown in Section 6. The main reason is that the subcarriers with deep fadings will have low received SNR and large error probabilities. The clipping estimation then propagates the errors and yields degraded estimation for both clipping noise and data. In SIMO fading channels, multiple receptions over independently faded channels not only provide the diversity gain for the data error performance, but also achieve better estimation for the clipping noise. The proposed joint MRC and clipping mitigation method thus exploits this benefit. In Section 6, we will show that the SER performance gets close to the MLSD bound within five iterations even for very small IBOs.

In summary, the proposed joint MRC and clipping mitigation method can provide the near-MLSD error performance. It requires the knowledge about the transmitter nonlinearity as well as receiver-side modifications. Compared with the transparent receiver, the extra complexity is on the order of $\mathcal{O}(N \log N)$, which is far less than the complexity of MLSD. From the transmitter perspective, the joint MRC and clipping mitigation method has lower complexity than PWLS and optimal clipping schemes. In addition, it can achieve better coding gain, which will be shown in the following section. 


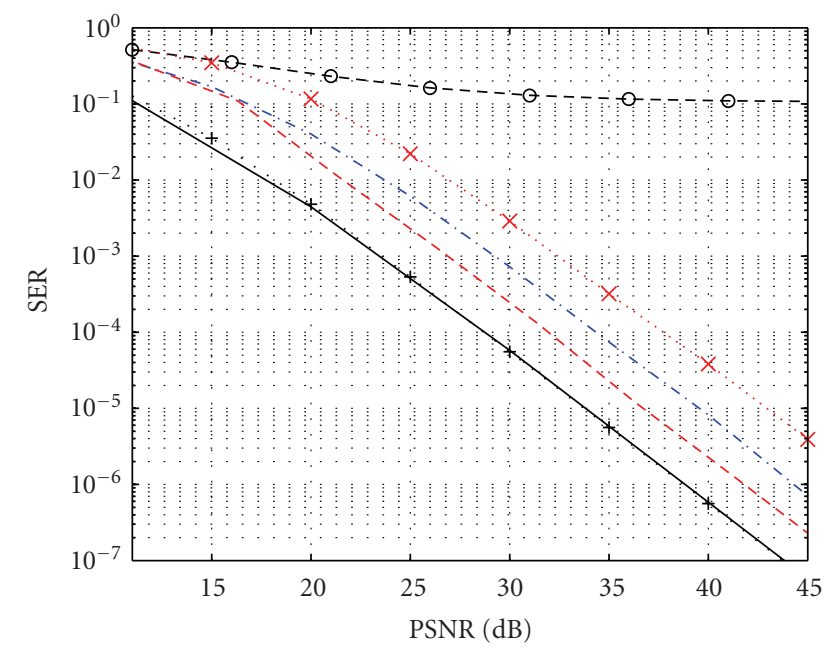

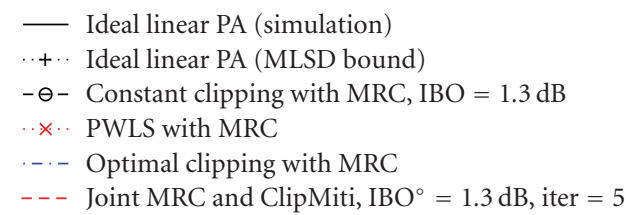

FIgURE 2: The SER versus PSNR curves for the constant clipping $(\mathrm{IBO}=1.3 \mathrm{~dB})$, PWLS, optimal clipping, joint MRC and clipping mitigation (with the optimal $\mathrm{IBO}^{\circ}=1.3 \mathrm{~dB}$ and five iterations) schemes, as well as the assumed ideal case with $\mathrm{IBO}=0 \mathrm{~dB}$ but no clipping. $N_{r}=2$.

\section{Simulation Results}

For all simulations in this section, the uncoded OFDM system has $N=512$ subcarriers and uses 16-QAM modulation. Unless otherwise specified, frequency-selective Rayleigh fading channel with two taps and $N_{r}=2$ receiving antennas are assumed. Since the antenna diversity is focused in this paper, the results are independent with the number of channel taps as long as the total average gain of these taps stays the same. In addition, ideal channel estimation is assumed so that $\mathbf{H}$ is known at the receivers.

In Figure 2, the SER versus PSNR curves are plotted for the proposed transceivers in the peak-power-limited SIMOOFDM channel.

First, the ideal case with IBO $=0 \mathrm{~dB}$ but linear PA (i.e., no clipping, thus $E\left[\left|y_{n}\right|^{2}\right]=P_{\text {peak }}$ and $\Delta(\mathrm{IBO})=$ 1 ) is plotted as a benchmark in Figure 2. Although only constant-envelope modulations (rather than OFDM) may actually achieve this error performance in practice, it gives an SER lower bound for this channel. For OFDM, by setting $\sigma_{s}^{2}=P_{\text {peak }}$ and assuming no clipping happens, Monte Carlo simulation gives the SER curve for this ideal case. The curve agrees well with the theoretical MLSD bound in (22) with $\mathrm{IBO}=0 \mathrm{~dB}$ and $\Delta(\mathrm{IBO})=1$.

Using the transparent receivers with the MRC weights given in Proposition 1, three transmitter schemes are also compared in Figure 2, namely, the constant clipping, the PWLS, and the optimal clipping approaches. As expected in Section 4, no antenna diversity can be obtained with the constant clipping method. In fact, the SER reaches an

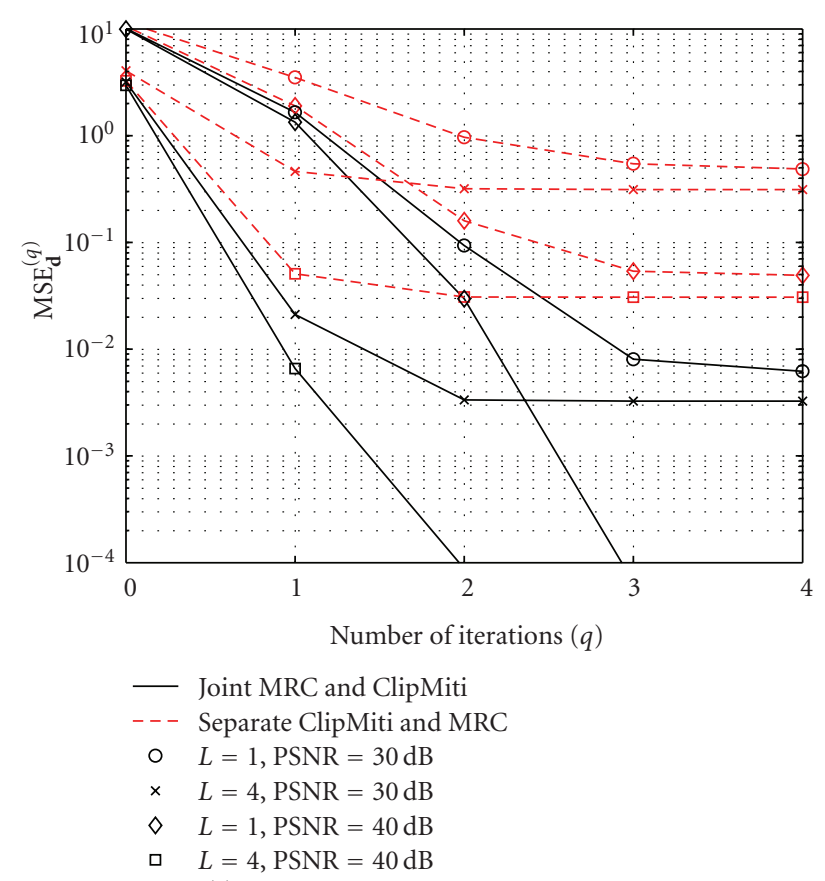

Figure 3: $\operatorname{MSE}_{\mathbf{d}}^{(q)}$ versus the number of iterations $(q)$ for the joint MRC and clipping mitigation methods. The corresponding MSE curves of separately using clipping mitigation [29] and MRC methods are also plotted for comparison. IBO $=1 \mathrm{~dB}, N_{r}=2$, the oversampling ratio $L=1$ or 4 , and PSNR $=30 \mathrm{~dB}$ or $40 \mathrm{~dB}$.

error floor that is determined by the clipping threshold. The PWLS-based transceiver can provide full antenna diversity but poor coding gain. Compared to the case with ideal linear PA, the PSNR degradation $\left(E\left[\mathrm{PAR}^{-1}\right]\right)^{-1}$ is more than $9 \mathrm{~dB}$ in the simulated system, as shown in Figure 2. On the other hand, the optimal clipping method achieves about $3 \mathrm{~dB}$ coding gain better than PWLS.

For the iterative method of (31), the MSE curves for the estimate of $\mathbf{d}$ (i.e., (32)) are plotted in Figure 3. The cases with PSNR $=30 \mathrm{~dB}$ and $40 \mathrm{~dB}$ as well as two oversampling ratios ( $L=1$ and 4$)$ are examined. The results illustrate that the MSE decreases quickly along with iterations, especially at high PSNR. For comparison, the corresponding MSE curves are plotted when the SISO iterative clipping mitigation method [29] is adopted on one of the antennas and the combining technique is used subsequently. It demonstrates that the benefit of multiple receiving antennas can be exploited to improve the clipping noise estimation performance. In Figure 4, the joint MRC and clipping mitigation method is illustrated to achieve nearMLSD SER performance within five iterations for both the Nyquist-rate and oversampled $(L=4)$ OFDM signals. It also works well for more than 2 receiving antennas as shown in Figure 5. In contrast, if the SISO iterative clipping mitigation method [29] and MRC are used separately, the antenna diversity cannot be collected even after 100 iterations.

As mentioned in (33), the optimal $\mathrm{IBO}^{\circ}$ can be determined to achieve the best SER for the joint MRC and clipping mitigation method. Some numerical results of the SER versus IBO curves are given for different PSNR values 


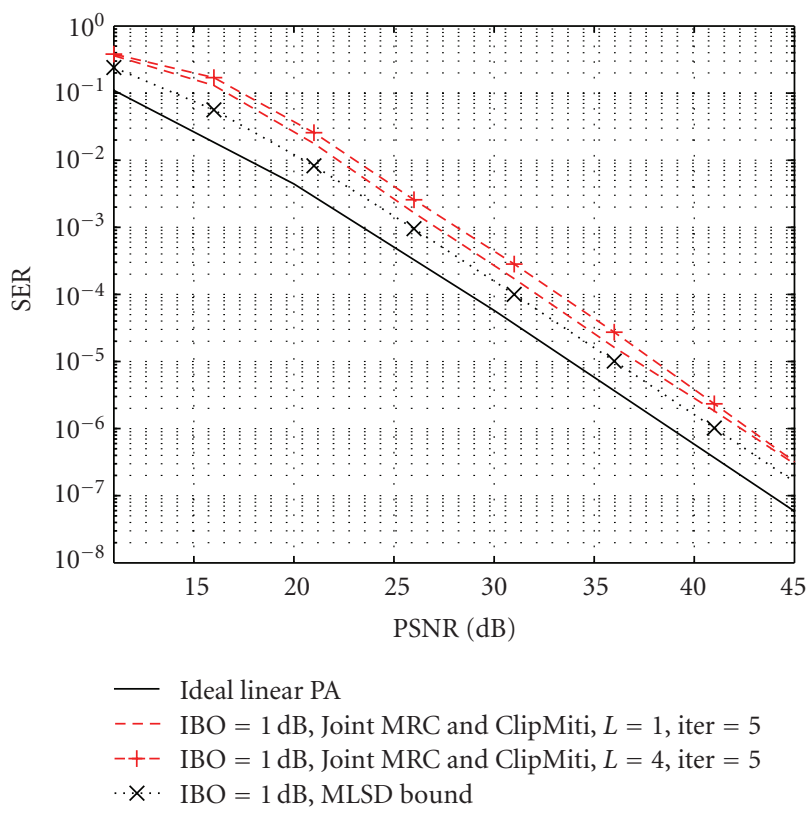

FIGURE 4: SER performance of the joint MRC and clipping mitigation method for both the Nyquist-rate and oversampling OFDM system. The SER curves of the ideal linear PA and the MLSD bound in (22) with IBO $=1 \mathrm{~dB}$ are also shown for comparison. $N_{r}=2$.

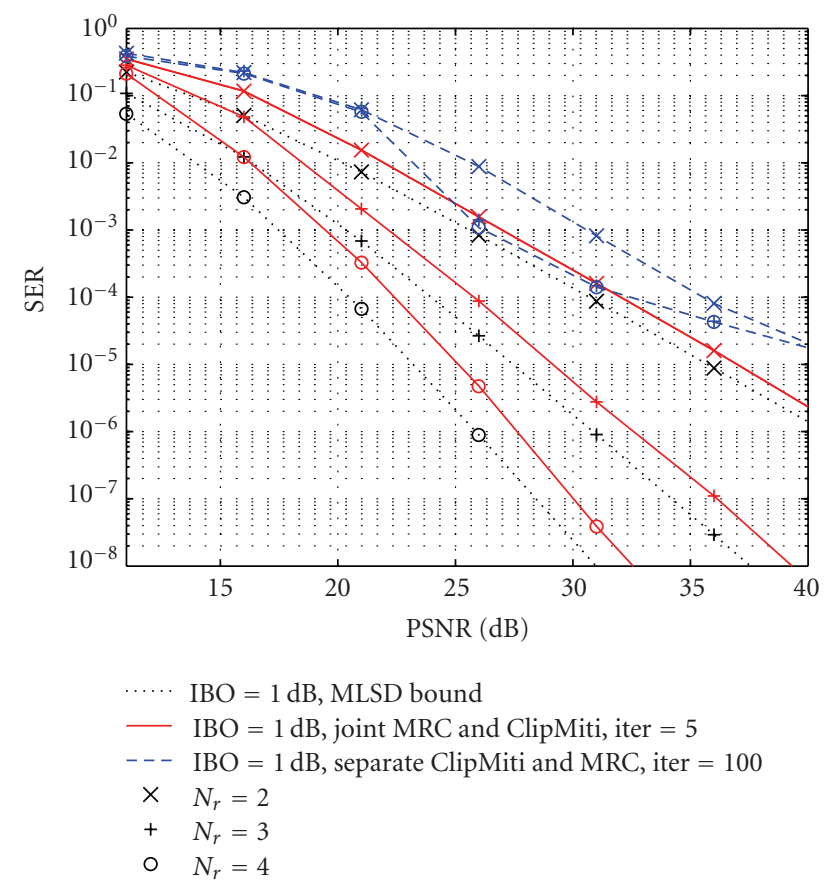

FIGURE 5: The SER versus PSNR curves for different numbers of receiving antennas $N_{r}=2$, 3, or 4 . The proposed joint MRC and clipping mitigation method achieves a near-MLSD SER within five iterations. But separately using clipping mitigation [29] and MRC cannot collect full antenna diversity even after 100 iterations. IBO $=1 \mathrm{~dB}$.

and numbers of antennas in Figure 6. The optimal IBO is found to remain about the same for different numbers of antennas. In addition, since diversity gain is achieved, $\mathrm{IBO}^{\circ}$ is generally independent with the PSNR. For example, $\mathrm{IBO}^{\circ} \approx$ $1.3 \mathrm{~dB}$ can be found for $N_{r}=2,3$, and 4 receiving antennas. With $\mathrm{IBO}^{\circ}=1.3 \mathrm{~dB}$ and five iterations, the SER curve for the joint MRC and clipping mitigation method is plotted back into Figure 2 and shown to outperform the other approaches.

\section{Conclusion}

In this paper, we have examined the antenna diversity gain in the peak-power-limited SIMO-OFDM system. The main conclusion is that full antenna diversity can be achieved for the transparent receiver by intelligently choosing the transmission method: PWLS and optimal clipping achieve diversity, while a constant back-off clipping does not. To 


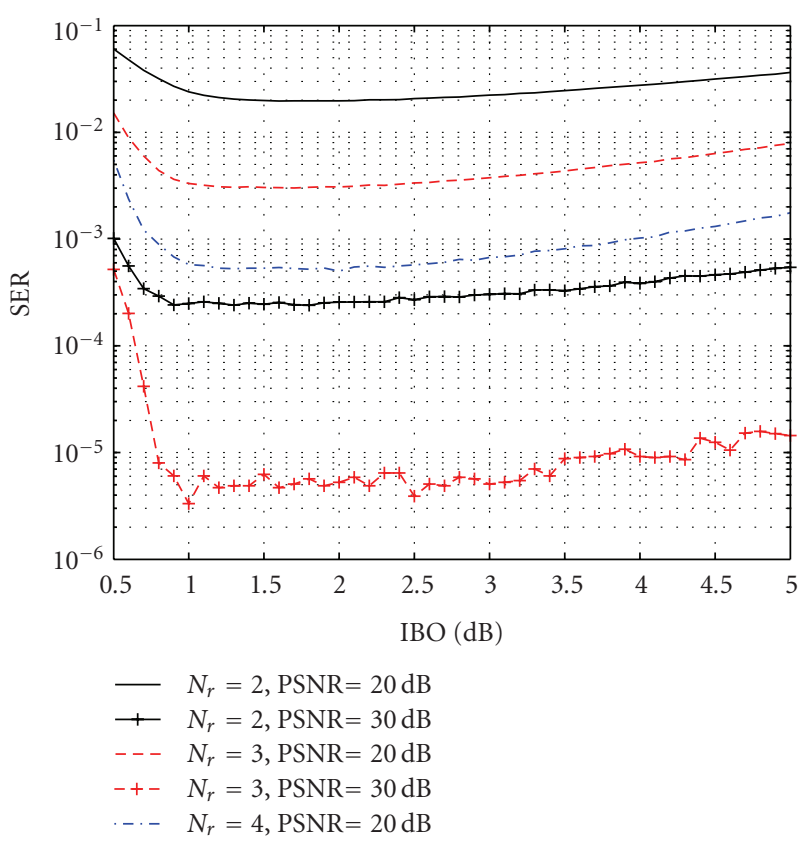

FIGURE 6: For PSNR $=20 \mathrm{~dB}$ or $30 \mathrm{~dB}$, the SER versus IBO curves for the joint MRC and clipping mitigation method with $N_{r}=2,3$, or 4 receiving antennas and 5 iterations.

achieve full antenna diversity, the MRC coefficients are derived for the peak-power-limited channel and can be obtained in the same way with those in the average-powerconstrained linear channel. Additionally, we showed that for systems where the receiver has perfect knowledge of the transmitter nonlinearity, antenna diversity can be achieved with low-complexity linear equalizers. The joint MRC and clipping mitigation method is also proposed to employ the multiple antennas to better estimate both the clipping noise and the data. To extend the results to coded multiantenna OFDM systems is a part of our future work.

\section{Appendices}

\section{A. Proof of Proposition 1}

The optimal MRC weights suffice to maximize the SNDR in (14). Taking the first-order derivative of $\mathrm{SNDR}_{k}$ with respect to $c_{k}$ and setting it to zero, we obtain

$$
\begin{aligned}
\frac{\partial}{\partial \mathbf{c}_{k}} \operatorname{SNDR}_{k}= & \frac{\left(\mathbf{c}_{k}^{T} \mathbf{h}_{k}^{\prime}\right)^{*} \sigma_{s}^{2} \mathbf{h}_{k}^{\prime}}{\left|\mathbf{c}_{k}^{T} \mathbf{h}_{k}\right|^{2} \sigma_{v}^{2}+\mathbf{c}_{k}^{H} \mathbf{c}_{k} \sigma_{w}^{2}} \\
& -\frac{\left|\mathbf{c}_{k}^{T} \mathbf{h}_{k}^{\prime}\right|^{2} \sigma_{s}^{2}\left(\left(\mathbf{c}_{k}^{T} \mathbf{h}_{k}\right)^{*} \sigma_{v}^{2} \mathbf{h}_{k}+\mathbf{c}_{k}^{*} \sigma_{w}^{2}\right)}{\left(\left|\mathbf{c}_{k}^{T} \mathbf{h}_{k}\right|^{2} \sigma_{v}^{2}+\mathbf{c}_{k}^{H} \mathbf{c}_{k} \sigma_{w}^{2}\right)^{2}}=0
\end{aligned}
$$

Recall that $\mathbf{h}_{k}^{\prime}=\alpha \mathbf{h}_{k}$. After some basic algebraic manipulations, (A.1) leads to

$$
\mathbf{c}_{k}^{T} \mathbf{h}_{k}^{\prime} \mathbf{c}_{k}^{*}=\mathbf{c}_{k}^{H} \mathbf{c}_{k} \mathbf{h}_{k}^{\prime} .
$$

Obviously, $\mathbf{c}_{k}=\mathbf{h}_{k}^{\prime *} / \mathbf{h}_{k}^{\prime H} \mathbf{h}_{k}^{\prime}=\mathbf{h}_{k}^{*} / \alpha \mathbf{h}_{k}^{H} \mathbf{h}_{k}$ satisfies (A.2). In addition, these weights are channel-normalizing (i.e., $\mathbf{c}_{k}^{T} \mathbf{h}_{k}^{\prime}=$ 1) as well as orthogonal to the channels of other subcarriers (i.e., $\mathbf{c}_{k}^{T} \mathbf{h}_{l}^{\prime}=0$, for all $k \neq l$ ). Therefore, $\mathbf{C}=\left[\mathbf{c}_{0}, \ldots, \mathbf{c}_{N-1}\right]$ with $\mathbf{c}_{k}=\mathbf{h}_{k}^{*} / \alpha \mathbf{h}_{k}^{H} \mathbf{h}_{k}$ gives the optimal MRC weights and the transparent receiver can decode according to $\widehat{\mathbf{s}}=\left\langle\mathbf{C}^{T} \mathbf{r}\right\rangle$.

\section{B. Proof of Proposition 2}

For transparent receivers, the SER performance is a function of the SNDR. Therefore, a necessary condition to achieve the diversity gain is that the postprocessing SNDR goes to infinity along with the PSNR. With the optimal MRC weights given in Proposition 1, the postprocessing SNDR becomes

$$
\mathrm{SNDR}_{k}=\frac{\mathbf{h}_{k}^{H} \mathbf{h}_{k}|\alpha|^{2} \sigma_{s}^{2}}{\mathbf{h}_{k}^{H} \mathbf{h}_{k} \sigma_{v}^{2}+\sigma_{w}^{2}} .
$$

For a given peak-power limit $P_{\text {peak }}$, increasing PSNR is equivalent to decreasing the noise power $\sigma_{w}^{2}$. From (B.1), we have

$$
\lim _{\sigma_{w}^{2} \rightarrow 0} \operatorname{SNDR}_{k}=\lim _{\sigma_{w}^{2} \rightarrow 0} \frac{|\alpha|^{2} \sigma_{s}^{2}}{\sigma_{v}^{2}} .
$$

As mentioned in Section $4,|\alpha| \leq 1$. In addition, $\sigma_{s}^{2} \leq P_{\text {peak }}$. Therefore, $\lim _{\sigma_{w}^{2} \rightarrow 0} \sigma_{v}^{2}=0$ is the necessary condition for the limit of SNDR in (B.2) to go to infinity, as well as for the transparent receiver to collect antenna diversity.

On the other hand, when $\lim _{\sigma_{w}^{2} \rightarrow 0} \sigma_{v}^{2}=0$, the limit of SNDR becomes

$$
\lim _{\sigma_{w}^{2} \rightarrow 0} \mathrm{SNDR}_{k}=\lim _{\sigma_{w}^{2} \rightarrow 0} \mathbf{h}_{k}^{H} \mathbf{h}_{k} \mathrm{SNR}
$$

which is the same as the postprocessing SNR of the linear channel case in Section 3. Plugging the SER of $P_{e}(\mathrm{PSNR})=$ $P_{s}(\mathrm{PSNR} / \mathrm{IBO})$ into the diversity gain definition of (7), full antenna diversity can be easily proved. For given $P_{\text {peak }}$ and IBO, by referring to (11), we have

$$
\begin{aligned}
G_{d} & =\lim _{\mathrm{PSNR} \rightarrow \infty}-\frac{\log P_{s}(\mathrm{PSNR} / \mathrm{IBO})}{\log \mathrm{PSNR}} \\
& =\lim _{\mathrm{PSNR}^{\prime} \rightarrow \infty}-\frac{\log P_{s}\left(\mathrm{PSNR}^{\prime}\right)}{\log \mathrm{PSNR}^{\prime}+\log \mathrm{IBO}}=N_{r},
\end{aligned}
$$

where $\mathrm{PSNR}^{\prime}=$ PSNR/IBO.

Therefore, for a fixed $P_{\text {peak }}$, the necessary and sufficient condition for the transparent receiver to collect full antenna diversity is that the distortion noise power vanishes as the PSNR increases. 


\section{Proof of Proposition 3}

Suppose that the symbol transmitted on the $k$ th subcarrier is $s_{k}$, but at the receiver it is erroneously decoded as $s_{k}^{\prime} \neq s_{k}$. The pairwise error probability is given as [30]

$$
\operatorname{Pr}\left(s_{k} \longrightarrow s_{k}^{\prime} \mid \mathscr{H}\right)=Q\left(\sqrt{\frac{\left|e_{k}\right|^{2}}{2 \sigma_{w}^{2} \Omega_{k k}}}\right),
$$

where $e_{k}=s_{k}-s_{k}^{\prime}$ and $\Omega_{k k}$ is the $(k, k)$ th element of

$$
\boldsymbol{\Omega}=\left(\mathscr{H}^{H} \mathscr{H}\right)^{-1}=\left(\mathbf{F} \boldsymbol{\Lambda} \mathbf{F}^{H} \mathbf{H}^{H} \mathbf{H} \mathbf{F} \boldsymbol{\Lambda} \mathbf{F}^{H}\right)^{-1} .
$$

Because the channel matrix $\mathbf{H}$ has full column rank with probability 1 and $\Lambda$ is a diagonal matrix with positive real diagonal entries, we have $\boldsymbol{\Omega}=\boldsymbol{\Gamma}\left(\mathbf{H}^{H} \mathbf{H}\right)^{-1} \boldsymbol{\Gamma}^{H}$, where $\boldsymbol{\Gamma}=$ $\left(\mathbf{F} \mathbf{\Lambda} \mathbf{F}^{H}\right)^{-1}$ is a nonsingular Hermitian and Toeplitz matrix. Since $\mathbf{H}^{H} \mathbf{H}=\operatorname{diag}\left(\left[\sum_{i=1}^{N_{r}}\left|H_{i, 0}\right|^{2}, \ldots, \sum_{i=1}^{N_{r}}\left|H_{i, N-1}\right|^{2}\right]\right), \Omega_{k k}$ can be expressed as

$$
\Omega_{k k}=\sum_{l=0}^{N-1} \frac{\left|\Gamma_{k, l}\right|^{2}}{\sum_{i=1}^{N_{r}}\left|H_{i, l}\right|^{2}} .
$$

Since $\Gamma$ has full rank, $\left\{l|| \Gamma_{k, l} \mid \neq 0\right\} \neq \varnothing$ for all $k$. Let $p \in$ $\left\{l|| \Gamma_{k, l} \mid \neq 0\right\}$ and $q=\arg \min _{l} \sum_{i=1}^{N_{r}}\left|H_{i, l}\right|^{2}$. We have the following inequalities

$$
a\left(\sum_{i=1}^{N_{r}}\left|H_{i, p}\right|^{2}\right)^{-1} \leq \Omega_{k k} \leq b\left(\sum_{i=1}^{N_{r}}\left|H_{i, q}\right|^{2}\right)^{-1},
$$

where $a \triangleq\left|\Gamma_{k, p}\right|^{2}$ and $b \triangleq \sum_{l=0}^{N-1}\left|\Gamma_{k, l}\right|^{2}$. Therefore, the bounds for the error probability are

$$
\begin{aligned}
Q\left(\sqrt{\frac{\left|e_{k}\right|^{2} \sum_{i=1}^{N_{r}}\left|H_{i, p}\right|^{2}}{2 a \sigma_{w}^{2}}}\right) & \leq \operatorname{Pr}\left(s_{k} \longrightarrow s_{k}^{\prime} \mid \mathscr{H}\right) \\
& \leq Q\left(\sqrt{\frac{\left|e_{k}\right|^{2} \sum_{i=1}^{N_{r}}\left|H_{i, q}\right|^{2}}{2 b \sigma_{w}^{2}}}\right) .
\end{aligned}
$$

Because the channel responses are complex Gaussian distributed, $\sum_{i=1}^{N_{r}}\left|H_{i, p}\right|^{2}$ is a chi-squared random variable with $2 N_{r}$ degrees of freedom. Therefore, by averaging over this random variable, the quantity on the left-hand side of (C.5) obeys

$$
E_{\mathbf{H}}\left[Q\left(\sqrt{\frac{\left|e_{k}\right|^{2} \sum_{i=1}^{N_{r}}\left|H_{i, p}\right|^{2}}{2 a \sigma_{w}^{2}}}\right)\right] \geq \beta_{1}(\mathrm{SNR})^{-N_{r}},
$$

where SNR $=\sigma_{s}^{2} / \sigma_{w}^{2}=\left((M-1) / 6 \sigma_{w}^{2}\right) d_{\min }^{2}$ for $M$-ary QAM constellations $\left(d_{\min }\right.$ is the minimum Euclidean distance of the constellation) and $\beta_{1}$ is a constant that is independent of the SNR. For the right-hand side (RHS) of (C.5), we have [30, Lemma 1]

$$
\operatorname{Pr}\left(\sum_{i=1}^{N_{r}}\left|H_{i, q}\right|^{2}<\xi\right) \leq N\left(\frac{\xi}{2}\right)^{N_{r}}, \quad \forall \xi \geq 0 .
$$

Integrating the RHS of (C.5) over the channel response gives

$$
\begin{gathered}
E_{\mathbf{H}}\left[Q\left(\sqrt{\frac{\left|e_{k}\right|^{2} \sum_{i=1}^{N_{r}}\left|H_{i, q}\right|^{2}}{2 b \sigma_{w}^{2}}}\right)\right] \\
=E_{\mathbf{H}}\left[\frac{1}{2} \operatorname{Pr}\left(\sum_{i=1}^{N_{r}}\left|H_{i, q}\right|^{2}<\frac{2 b \sigma_{w}^{2} \epsilon^{2}}{\left|e_{k}\right|^{2}}\right)\right] \\
\leq E_{\epsilon}\left[\frac{N}{2}\left(\frac{b \sigma_{w}^{2} \epsilon^{2}}{d_{\min }^{2}}\right)^{N_{r}}\right]=\beta_{2}(\mathrm{SNR})^{-N_{r}},
\end{gathered}
$$

where $\epsilon$ is a Gaussian random variable with zero mean and unit variance and $\beta_{2}$ is a constant independent of the SNR. Therefore, combining (C.5), (C.6), and (C.8), we infer

$$
\beta_{1}(\mathrm{SNR})^{-N_{r}} \leq P_{s}=E_{\mathrm{H}}\left[\operatorname{Pr}\left(s_{k} \longrightarrow s_{k}^{\prime} \mid \mathcal{H}\right)\right] \leq \beta_{2}(\mathrm{SNR})^{-N_{r}},
$$

which means the diversity order collected by the ZF equalizer with known $\Lambda$ is $N_{r}$.

\section{Acknowledgment}

This work was supported in part by the U. S. Army Research Laboratory under the Collaborative Technology Alliance Program, Cooperative Agreement DAAD19-01-2-0011.

\section{References}

[1] "Wireless LAN Medium Access Control (MAC) and Physical Layer (PHY) Specifications: High-Speed Physical Layer in the 5 GHz Band," IEEE Std. 802.11a, September 1999.

[2] "IEEE Standard for Local and Metropolitan Area Networks Part 16: Air Interface for Fixed Broadband Wireless Access Systems," IEEE Std. 802.16-2004 (Revision of IEEE Std. 802.16-2001), 2004.

[3] G. L. Stüber, J. R. Barry, S. W. Mclaughlin, Y. E. Li, M. A. Ingram, and T. G. Pratt, "Broadband MIMO-OFDM wireless communications," Proceedings of the IEEE, vol. 92, no. 2, pp. 271-293, 2004.

[4] H. Ekström, A. Furuskär, J. Karlsson, et al., "Technical solutions for the 3G long-term evolution," IEEE Communications Magazine, vol. 44, no. 3, pp. 38-45, 2006.

[5] J. Tellado, Multicarrier Modulation with Low PAR: Applications to DSL and Wireless, Kluwer Academic Publishers, Norwell, Mass, USA, 2000.

[6] S. H. Han and J. H. Lee, "An overview of peak-to-average power ratio reduction techniques for multicarrier transmission," IEEE Wireless Communications, vol. 12, no. 2, pp. 56-65, 2005.

[7] J. G. Proakis, Digital Communications, McGraw-Hill, New York, NY, USA, 4th edition, 2001. 
[8] D. Tse and P. Viswanath, Fundamentals of Wireless Communications, Cambridge University Press, Cambridge, UK, 2005.

[9] F. Peng and W. E. Ryan, "MLSD bounds and receiver designs for clipped OFDM channels," IEEE Transactions on Wireless Communications, vol. 7, no. 9, pp. 3568-3578, 2008.

[10] G. L. Stüber, Principles of Mobile Communication, Kluwer Academic Publishers, Norwell, Mass, USA, 2nd edition, 2001.

[11] G. J. Foschini and M. J. Gans, "On limits of wireless communications in a fading environment when using multiple antennas," Wireless Personal Communications, vol. 6, no. 3, pp. 311-335, 1998.

[12] U.-K. Kwon, D. Kim, K. Kim, and G.-H. Im, "Amplitude clipping and iterative reconstruction of STBC/SFBC-OFDM signals," IEEE Signal Processing Letters, vol. 14, no. 11, pp. 808811, 2007.

[13] S. Bittner, P. Zillmann, and G. Fettweis, "Iterative correction of clipped and filtered spatially multiplexed OFDM signals," in Proceedings of IEEE Vehicular Technology Conference (VTC '08), pp. 953-957, May 2008.

[14] U.-K. Kwon, D. Kim, and G.-H. Im, "Amplitude clipping and iterative reconstruction of MIMO-OFDM signals with optimum equalization," IEEE Transactions on Wireless Communications, vol. 8, no. 1, pp. 268-277, 2009.

[15] R. J. Baxley, C. Zhao, and G. T. Zhou, "Constrained clipping for crest factor reduction in OFDM," IEEE Transactions on Broadcasting, vol. 52, no. 4, pp. 570-575, 2006.

[16] J. Armstrong, "Peak-to-average power reduction for OFDM by repeated clipping and frequency domain filtering," Electronics Letters, vol. 38, no. 5, pp. 246-247, 2002.

[17] X. Ma and W. Zhang, "Fundamental limits of linear equalizers: diversity, capacity, and complexity," IEEE Transactions on Information Theory, vol. 54, no. 8, pp. 3442-3456, 2008.

[18] J. Lu, T. T. Tjhung, and C. C. Chai, "Error probability performance of L-branch diversity reception of MQAM in Rayleigh fading," IEEE Transactions on Communications, vol. 46, no. 2, pp. 179-181, 1998.

[19] H. E. Rowe, "Memoryless nonlinearities with gaussian inputs: elementary results," The Bell System technical journal, vol. 61, no. 7, pp. 1519-1525, 1982.

[20] R. Raich, H. Qian, and G. T. Zhou, "Optimization of SNDR for amplitude-limited nonlinearities," IEEE Transactions on Communications, vol. 53, no. 11, pp. 1964-1972, 2005.

[21] H. Ochiai, "Performance analysis of peak power and bandlimited OFDM system with linear scaling," IEEE Transactions on Wireless Communications, vol. 2, no. 5, pp. 1055-1065, 2003.

[22] S. Litsyn and A. Shpunt, "A balancing method for PMEPR reduction in OFDM signals," IEEE Transactions on Communications, vol. 55, no. 4, pp. 683-691, 2007.

[23] R. W. Bäuml, R. F. H. Fischer, and J. B. Huber, "Reducing the peak-to-average power ratio of multicarrier modulation by selected mapping," Electronics Letters, vol. 32, no. 22, pp. 2056-2057, 1996.

[24] H. Qian, R. Raich, and G. T. Zhou, "Optimization of sndr in the presence of amplitude limited nonlinearity and multipath fading," in Proceedings of the Asilomar Conference on Signals, Systems and Computers (ACSSC '04), vol. 1, pp. 712-716, Pacific Grove, Calif, USA, November 2004.

[25] F. Peng and W. E. Ryan, "New approaches to clipped OFDM channels: modeling and receiver design," in Proceedings of IEEE Global Telecommunications Conference (GLOBECOM '05), vol. 3, pp. 1490-1494, St. Louis, Mo, USA, December 2005.
[26] D. Declercq and G. B. Giannakis, "Recovering clipped OFDM symbols with Bayesian inference," in Proceedings IEEE International Conference on Acoustics, Speech and Signal Processing (ICASSP '00), vol. 1, pp. 157-160, Istanbul, Turkey, June 2000.

[27] F. Peng and W. E. Ryan, "On the capacity of clipped OFDM channels," in Proceedings of IEEE International Symposium on Information Theory (ISIT '06), pp. 1866-1870, Seattle, Wash, USA, July 2006.

[28] T. N. E. Greville, "Note on the generalized inverse of a matrix product," SIAM Review, vol. 8, no. 4, pp. 518-521, 1966.

[29] J. Tellado, L. M.C. Hoo, and J. M. Cioffi, "Maximumlikelihood detection of nonlinearly distorted multicarrier symbols by iterative decoding," IEEE Transactions on Communications, vol. 51, no. 2, pp. 218-228, 2003.

[30] X. Ma, W. Zhang, and A. Swami, "Lattice-reduction aided equalization for OFDM systems," IEEE Transactions on Wireless Communications, vol. 8, no. 4, pp. 1608-1613, 2009. 